\title{
High CCL5 expression is associated with osteosarcoma metastasis and poor prognosis of patients with osteosarcoma
}

\author{
KAI SUN ${ }^{1 *}$, CHEN GONG $^{2 *}$, HAO PENG $^{1}$, HONGSONG FANG $^{1}$, JIANLIN ZHOU ${ }^{1}$, \\ JIANPING LI ${ }^{1}$, SEN CHEN ${ }^{1}$ and HUIFENG ZHENG ${ }^{1}$ \\ ${ }^{1}$ Department of Orthopedics, Renmin Hospital of Wuhan University, Wuhan, Hubei 430060; \\ ${ }^{2}$ Department of Oncology, Tongji Hospital, Tongji Medical College, Huazhong University \\ of Science and Technology, Wuhan, Hubei 430030, P.R. China
}

Received March 27, 2016; Accepted March 2, 2017

DOI: $10.3892 / \mathrm{mmr} .2017 .7458$

\begin{abstract}
Osteosarcoma is the most common primary malignant tumor of the skeletal system and is characterized by an aggressive clinical course and high metastatic potential. Regulated upon Activation Normal T cell Expressed and Secreted, also termed C-C motif chemokine ligand 5 (CCL5), is associated with metastasis and poor prognosis in various types of cancer. The aim of the current study was to investigate the association between CCL5 expression and clinicopathological features and prognosis in patients with osteosarcoma. Tissue microarrays and reverse transcription-quantitative polymerase chain reaction and immunohistochemistry were used to examine the expression of CCL5 in human osteosarcoma tissues. The prognostic value of CCL5 expression was evaluated by the Kaplan-Meier method and Cox proportional hazards regression model. The rate of high CCL5 expression was significantly higher in metastatic osteosarcomas than in osteosarcomas without metastases. The overall survival rates $(\mathrm{P}=0.001)$ and the metastasis-free survival rates $(\mathrm{P}<0.001)$ of the low CCL5 expression group were significantly higher than the CCL5 high expression group. Multivariate Cox regression analysis indicated that CCL5 expression had independent predictive value for the prognosis of patients with osteosarcoma. In conclusion, the data of the current study indicated that CCL5 may serve as a biomarker for prognosis of osteosarcoma, and may be a potential molecular target for osteosarcoma therapy.
\end{abstract}

Correspondence to: Professor Hao Peng, Department of Orthopedics, Renmin Hospital of Wuhan University, 99 Zhang Zhi-dong Road, Wuhan, Hubei 430060, P.R. China

E-mail: penghaowhu@163.com

${ }^{*}$ Contributed equally

Key words: osteosarcoma, regulated upon activation normal $\mathrm{T}$ cell expressed and secreted, C-C motif chemokine ligand 5, metastasis, prognosis

\section{Introduction}

Osteosarcoma is the most common primary sarcoma of the skeletal system in adolescents and young adults (1). It is a major death-causing disease in adolescence, due to its rapid progression and poor prognosis. The major causes of death are distant metastasis, particularly pulmonary metastases. Due to lack of effective and efficient tumor biomarkers for early diagnosis, despite the rapid development in therapeutic methods, including surgery, chemotherapy, and radiation therapy, there has been minimal change for patients with metastatic disease and their long-term survival rate remains at $25-30 \%$ (2), the prognosis remains poor and the majority of patients die at the advanced stages. Although many immunohistochemical markers and genetic proteins have been studied, there is an urgent need to develop new targets for the diagnosis, treatment and prognosis of osteosarcoma.

Regulated upon Activation Normal T cell Expressed and Secreted also termed C-C motif chemokine ligand 5 (CCL5), was initially described for its role in inflammatory diseases; mediating chemotactic activity in T cells, natural killer cells, monocytes, dendritic cells, eosinophils and basophils (3-8). CCL5 is associated with chronic inflammatory disease and cancer (9). The association between CCL5 expression and cancer has been reported in lung and prostate cancer $(10,11)$. CCL5 is a marker of poor outcomes in patients with breast, cervical, prostate, gastric or pancreatic cancer (11-15). Certain studies have indicated that CCL5 is associated with cell migration and invasion in several cancer cell lines $(9,16)$.

However, the expression of CCL5 in human osteosarcoma, and its clinicopathological and prognostic significance has not been established. In the present study, reverse transcription-quantitative polymerase chain reaction (RT-qPCR) and immunohistochemistry (IHC) were used to examine the expression of CCL5 in human osteosarcoma tissues. The correlations between CCL5 expression and patient clinicopathologic and prognostic significance were assessed to determine whether the expression of CCL5 could be used as a metastasis or prognostic biomarker for patients with osteosarcoma. 


\section{Materials and methods}

Patients and tissue specimens. The study was approved by the ethics committee of Renmin Hospital of Wuhan University (Wuhan, China). Written informed consent was obtained from all patients prior to enrollment in the present study. From January 2012 to January 2014, 28 fresh osteosarcoma tissue samples were obtained from 15 patients with primary osteosarcoma with no metastases and 13 patients with metastases in Renmin Hospital of Wuhan University. No patients had received anticancer treatment prior to surgery. Paraffin-embedded pathological specimens taken from 90 patients with osteosarcoma between January 2003 to January 2015 were obtained from the Department of Orthopedics, Renmin Hospital of Wuhan University. Clinicopathological characteristics for these patients, including gender, age, histological subtype, Enneking staging and metastasis are described in Table I. No patient had received radiotherapy or chemotherapy before surgery.

$R T-q P C R$. Total RNA was extracted using TRIzol (Invitrogen; Thermo Fisher Scientific, Inc., Waltham, MA, USA) following the standard protocol and purified with the RNeasy Plus Mini kit (Qiagen Gmbh, Hilden, Germany). SuperScript III kit (Invitrogen; Thermo Fisher Scientific, Inc.) was used to reverse transcribe cDNA. qPCR was performed using SYBR Green Reaction Mix (Applied Biosystems; Thermo Fisher Scientific, Inc.) and the StepOnePlus thermocycler (Applied Biosystems; Thermo Fisher Scientific, Inc.). The CCL5 primer sequences used are as follows: Forward, 5'-TGCCCACATCAAGGAGTA TTT-3'; and reverse, 5'-GATGTACTCCCGAACCCATTT-3'. The transcript level of $18 \mathrm{~S}$ rRNA was used as endogenous reference gene, with the primer sequences as follows: Forward, 5'-GTAACCCGTTGAACCCCATT-3'; and reverse, 5'-CCA TCCAATCGGTAGTAGCG-3'. Gene expression was analyzed by the comparative Cq method (17).

Tissue microarray (TMA). Tumor tissue samples from the 90 patients with osteosarcoma were collected, fixed in formalin, and embedded in paraffin. Hematoxylin and eosin-stained sections were used to define representative tumor regions without spontaneous necrosis. The formalin-fixed, paraffin-embedded two targeted core samples of each specimen were obtained from these representative regions using a tissue array instrument (MiniCore; ALPHELYS, Plaisir, France). Tissue cylinders with a diameter of $1.5 \mathrm{~mm}$ were punched and arrayed on a recipient paraffin block. Sections $(5 \mu \mathrm{m})$ of the tissue array (recipient) block were cut and placed on glass slides.

$I H C$. The tissue sections were deparaffinized in xylene and rehydrated in a series of graded alcohols. Hydrogen peroxide (3\%) was used to block endogenous peroxidase activity for 10 min. Slides were boiled in EDTA buffer ( $\mathrm{pH}$ 8.0) in a pressure cooker for $15 \mathrm{~min}$ for antigen retrieval, and then blocked with $10 \%$ normal goat serum for $30 \mathrm{~min}$ at room temperature. Subsequently, the sections were immunostained with CCL5 primary antibody (1:100 dilution; cat no. ab9679; Abcam, Cambridge, MA, USA) and incubated at $4^{\circ} \mathrm{C}$ overnight. After rinsing with PBS three times for $5 \mathrm{~min}$ each, the sections were incubated in goat anti-rabbit horseradish peroxidase-conjugated secondary antibody (1:2,000 dilution; cat no. ab205718;
Abcam) for $30 \mathrm{~min}$ at room temperature and stained with diaminobenzidine.

The staining results were determined using a semi-quantitative method by assessing the intensity of staining and the percentage of CCL5-positive cells. Staining intensity was categorized as follows: Negative, 0 ; weak, 1 , moderate, 2; and strong, 3 . The percentage of staining was scored as follows: $0,0 \%$ positive cells; $1,1-25 \%$ positive cells; $2,26-50 \%$ positive cells; $3,51-75 \%$ positive cells; or $4,>75 \%$ positive cells; according to the percentages of positively stained areas in relation to the whole carcinoma area (or entire section for normal samples). The sum of the staining intensity and extent scores was used as the final staining scores (0-7). Tumors with a final staining score of $>3$ were considered to be CCL5-positive. Cells were evaluated using an Olympus CKX41 inverted microscope under x200 magnification (Olympus Corporation, Tokyo, Japan). Expression for CCL5 was scored by two independent pathologists blinded to the clinicopathological data.

Statistical analysis. SPSS version 13.0 (SPSS, Inc., Chicago, IL, USA) was used for the analyses. The difference in CCL5 mRNA expression levels between metastatic osteosarcoma group and non-metastatic osteosarcoma group was analyzed using Student's t-test. The correlation of CCL5 expression and clinicopathological was evaluated by $\chi^{2}$ test. Univariate and multivariate analyses were performed with the Cox proportional hazard regression model to determine associations between clinicopathological variables and cancer-associated mortality. Kaplan-Meier method and log-rank test were used for survival analysis. $\mathrm{P}<0.05$ was considered to indicate a statistically significant difference.

\section{Results}

$R T$-qPCR analysis of CCL5 expression in osteosarcoma. RT-qPCR analysis revealed that the gene expression level of CCL5 was significantly increased in metastatic osteosarcoma group compared with the non-metastatic osteosarcoma group, indicating that CCL5 is highly active in osteosarcomas with metastases $(\mathrm{P}<0.05$; Fig. 1$)$.

Association between CCL5 expression and poor prognosis in patients with osteosarcoma. Immunohistochemistry was used to evaluate CCL5 expression in all 90 samples within the osteosarcoma TMA. Expression of CCL5 was predominantly localized to the cytoplasm and the membrane of cancer cells (Fig. 2). The high CCL5 expression rate in metastatic osteosarcomas $(80 \% ; 24 / 30)$ was significantly higher than the high CCL5 expression rate in non-metastatic osteosarcomas (26.7\%; 16/60; $\mathrm{P}<0.001)$. The high CCL5 expression rate in patients with Enneking staging I, II (58.8\%; 30/51) was significantly higher than the high CCL5 expression rate in Enneking staging III (25.6\%; 10/39; P<0.05). There were no significant associations between the expression of CCL5 and gender, age or histological subtype (Table I).

Univariate analysis was performed to evaluate associations between patient prognosis and several clinicopathological factors including CCL5 expression, gender, age, histological subtype (conventional: Osteoblastic, chondroblastic, fibroblastic osteosarcomas), Enneking staging and metastasis. The 
Table I. Association of CCL5 expression with patient clinicopathological features in osteosarcoma.

\begin{tabular}{|c|c|c|c|c|}
\hline Feature & $\begin{array}{c}\text { Total } \\
(\mathrm{n}=90)\end{array}$ & $\begin{array}{c}\text { CCL5 low } \\
\text { expression }(n=50)(\%)\end{array}$ & $\begin{array}{c}\text { CCL5 high } \\
\text { expression }(n=40)(\%)\end{array}$ & P-value \\
\hline Gender & & & & 0.064 \\
\hline Male & 51 & $24(47.1)$ & $27(52.9)$ & \\
\hline Female & 39 & $26(66.7)$ & $13(33.3)$ & \\
\hline Age & & & & 0.13 \\
\hline$<20$ years & 46 & $22(47.8)$ & $24(52.2)$ & \\
\hline$\geq 20$ years & 44 & $28(63.6)$ & $16(36.4)$ & \\
\hline Histological subtype & & & & 0.116 \\
\hline Special & 51 & $32(62.7)$ & $19(37.3)$ & \\
\hline Conventional & 39 & $18(46.2)$ & $21(53.8)$ & \\
\hline Enneking staging & & & & 0.002 \\
\hline $\mathrm{I}, \mathrm{II}$ & 39 & $29(74.4)$ & $10(25.6)$ & \\
\hline III & 51 & $21(41.2)$ & $30(58.8)$ & \\
\hline Metastasis & & & & $<0.001$ \\
\hline No & 60 & $44(73.3)$ & $16(26.7)$ & \\
\hline Yes & 30 & $6(20)$ & $24(80)$ & \\
\hline
\end{tabular}

CCL5, C-C motif chemokine ligand 5.

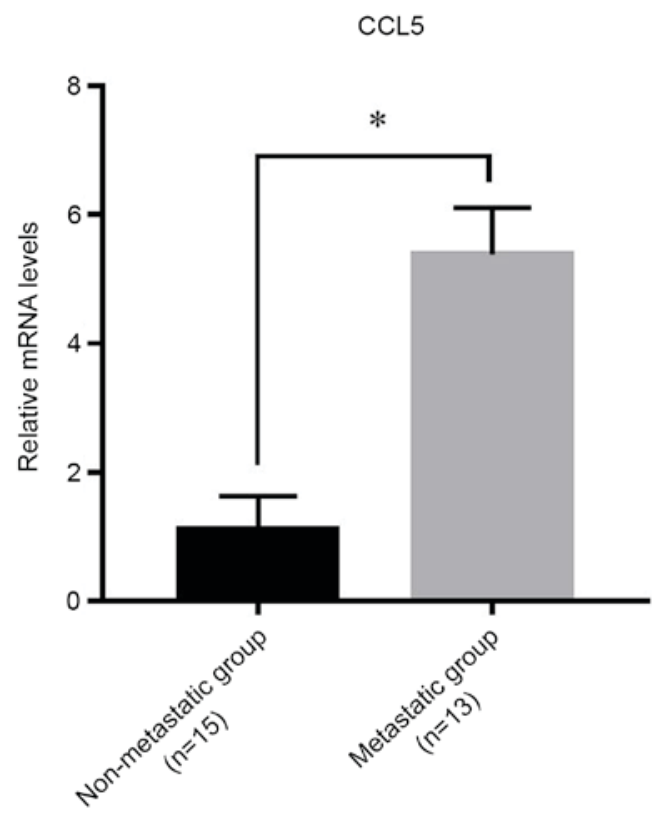

Figure 1. CCL5 expression in metastatic osteosarcoma group and non-metastatic osteosarcoma group. CCL5 mRNA levels were determined by reverse transcription-quantitative polymerase chain reaction. ${ }^{*} \mathrm{P}<0.05$, as indicated. CCL5, C-C motif chemokine ligand 5 .

results indicated that CCL5 expression, Enneking staging and metastasis were significantly associated with poorer prognosis (Table II).

Subsequently, multivariate analysis was performed to further understand the association between the clinicopathological factors and prognosis, indicated that CCL5 expression $(\mathrm{P}=0.001)$ was found to be statistically significant independent of Enneking staging and metastasisfor patients

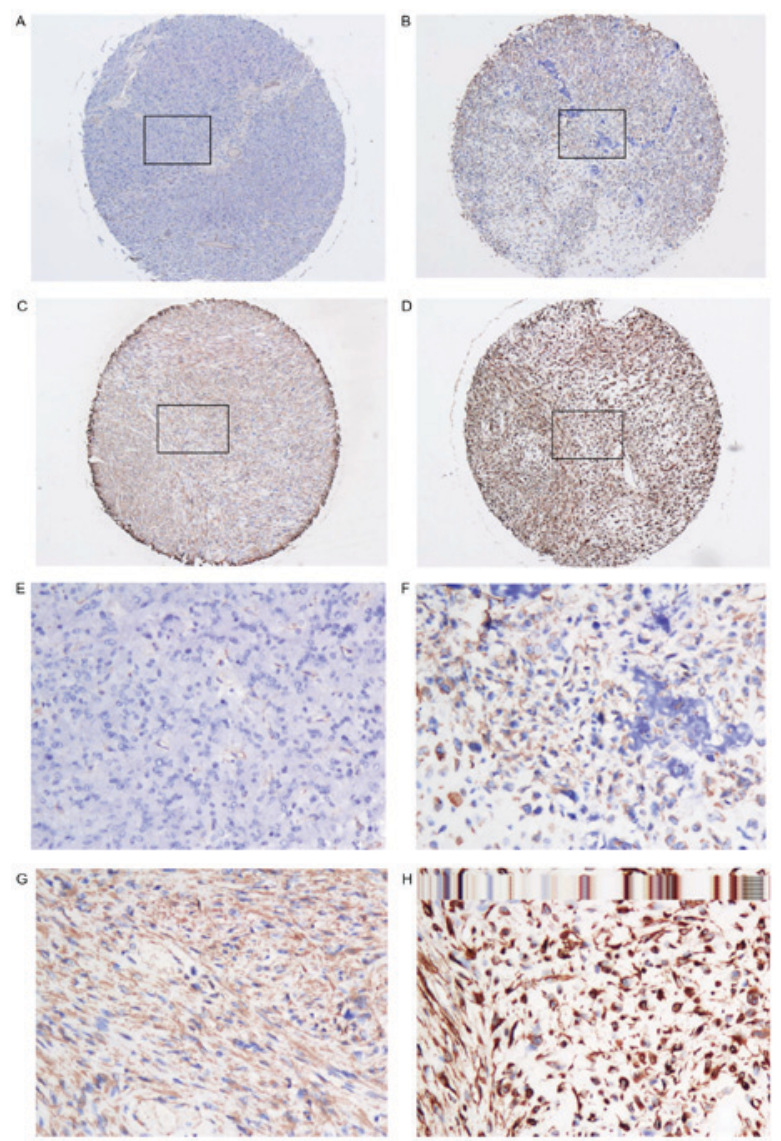

Figure 2. Representative immunohistochemical staining samples for negative, weak, moderate, strong CCL5 expression in osteosarcoma tissues. Upper panels: $x 40$ magnification of tissue sections at staining intensity levels of (A) 0, (B) 1, (C) 2, and (D) 3. Lower panels, x200 magnification at staining intensity levels of $(\mathrm{E}) 0,(\mathrm{~F}) 1,(\mathrm{G}) 2$, and $(\mathrm{H}) 3$. The boxed area in the upper $\mathrm{x} 40$ panels are shown at $\mathrm{x} 200$ in the lower panels. CCL5, C-C motif chemokine ligand 5. 
Table II. Cox proportional hazards model analysis of prognostic factors in patients with osteosarcoma.

\begin{tabular}{|c|c|c|c|c|}
\hline Feature & HR & $95 \% \mathrm{CI}$ & Unfavorable/favorable & P-value \\
\hline \multicolumn{5}{|l|}{ Univariate analysis } \\
\hline CCL5 & 2.894 & $1.504-5.571$ & High/low & 0.001 \\
\hline Gender & 1.027 & $0.546-1.931$ & Male/female & 0.934 \\
\hline Age, & 0.756 & $0.402-1.423$ & $\geq 20 /<20$ years & 0.386 \\
\hline Histological subtype & 0.826 & $0.436-1.565$ & Conventional/special & 0.558 \\
\hline Enneking staging & 2.245 & $1.130-4.461$ & III/I,II & 0.021 \\
\hline Metastasis & 2.46 & $1.287-4.701$ & Yes/no & 0.006 \\
\hline \multicolumn{5}{|l|}{ Multivariate analysis } \\
\hline CCL5 & 3.18 & $1.650-6.672$ & High/low & 0.001 \\
\hline Gender & 1.589 & $0.808-3.127$ & Male/female & 0.18 \\
\hline Age & 0.764 & $0.404-1.444$ & $\geq 20 /<20$ years & 0.40 \\
\hline Histologic subtype & 0.810 & $0.404-1.625$ & Conventional/special & 0.55 \\
\hline Enneking staging & 1.611 & $0.742-3.498$ & III/I,II & 0.228 \\
\hline Metastasis & 1.421 & $0.615-3.285$ & Yes/no & 0.411 \\
\hline
\end{tabular}

HR, hazard ratio; CI, confidence interval; CCL5, C-C motif chemokine ligand 5.

with osteosarcoma (Table II). The outcome indicated that CCL5 is a potential biomarker for evaluation of the prognoses of patients with osteosarcoma.

The overall survival $(\mathrm{P}=0.001)$ and the metastasis-free survival $(\mathrm{P}<0.001)$ rates of the CCL5 low expression group were significantly higher than the CCL5 high expression group, as demonstrated by Kaplan-Meier analyses and the log-rank test (Fig. 3).

\section{Discussion}

Osteosarcoma is the most common primary malignant tumor of the skeletal system and is characterized by aggressive clinical development and the high capacity for metastatic potential, a major cause of mortality (18). Improvements in chemotherapy and surgical advances have increased the 5-year overall survival rate of patients with localized disease to between 60 and $70 \%$; however, the prognosis remains generally poor, particularly for patients with metastasis, with the survival rate ranging from $11-20 \%$. The patients with metastasis have $<20 \%$ chance of long-term survival despite the use of advanced chemotherapeutic drugs (2). The clinical improvement appears to have reached a bottleneck. Therefore, it is urgent to find suitable metastasis and prognosis-associated biomarkers to evaluate the prognoses of patients with osteosarcoma.

CCL5 is a chemokine, and the primary role has been defined as a chemoattractant for immune cells. CCL5 was previously observed to have a significant effect on migration of numerous tumor types, and an important role in oncogenic processes (19). In prostate cancer, CCL5 may be part of an autocrine loop to produce multiple signals that affect cancer cell invasion and proliferation (11). In breast cancer, high CCL5 expression has been demonstrated to be demonstrated to have a role in poor prognosis (12). In lung cancer, a study suggested that CCL5 acts via phosphoinositide 3-kinase (PI3K)/Akt serine/threonine kinase, resulting in the activation of $\alpha v \beta 3$ integrin and facilitating
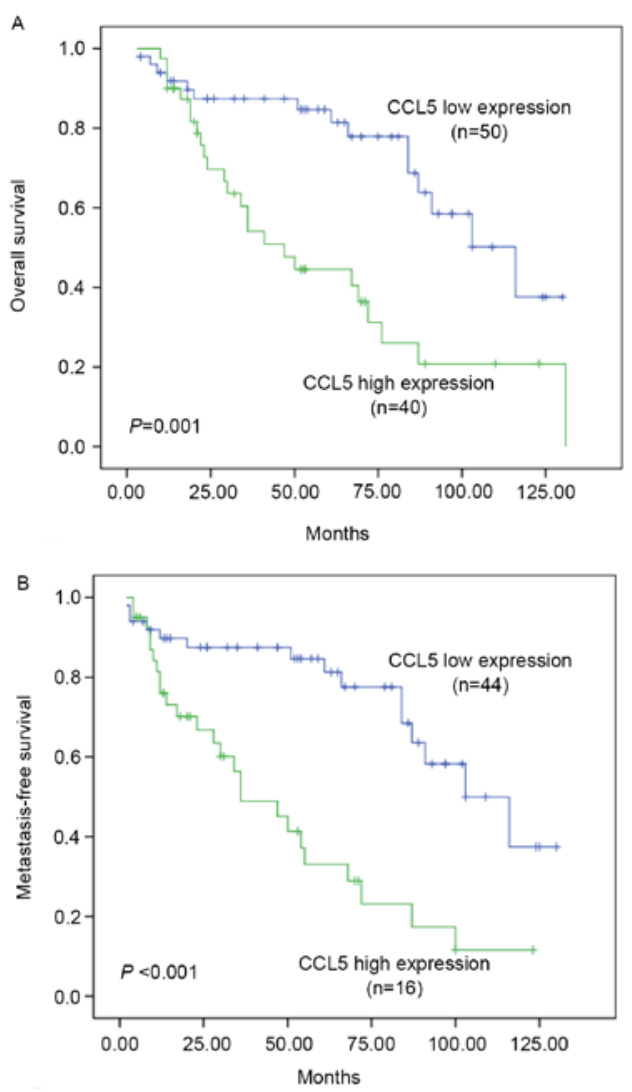

Figure 3. (A) Kaplan-Meier analysis of overall survival with high or low CCL5 expression ( $\mathrm{P}=0.001, \mathrm{log}$-rank test). (B) Kaplan-Meier analysis of metastasis-free survival with high or low CCL5 expression $(\mathrm{P}<0.001$, log-rank test). CCL5, C-C motif chemokine ligand 5.

the migration of human lung cancer cells (20). Other research has indicated that CCL5 is released by osteosarcoma cells and promotes tumor cells proliferation, invasion and migration (21). 
Wang et al (22) reported that there is a positive association between the expression of CCL5 and vascular endothelial growth factor (VEGF) in human osteosarcoma tissues, and the high level of CCL5 expression is correlated strongly with VEGF expression and tumor stage. The result of the previous study suggested that CCL5 may have important role in promoting VEGF-mediated tumor angiogenesis and metastatic progression of human osteosarcoma. Additionally, Wang et al (23) reported that PI3K and Akt are involved in CCL5-induced cell migration in osteosarcoma cells and that CCL5 can enhance cell migration in human osteosarcoma cell lines; the mechanisms involved an increase the expression of $\alpha v \beta 3$ integrin and activation of CCR 5 receptor via MEK, extracellular signal-regulated kinase and nuclear factor- $\kappa \mathrm{B}$ pathways. Matrix metalloproteinases (MMPs) are correlated with metastasis; Stormes et al (24) indicated that inhibition of tumor-derived CCL5 correlates with inhibition of metastasis and protease gene expression. The results indicated that CCL5 promotes MMP production in tumor cells, and tumor-derived CCL5 contributes to the metastatic potential. Therefore, the metastasis of tumor can be considered to be a final outcome of the cross linking of multiple factors.

The present study used RT-qPCR to examine the expression levels of CCL5 mRNA in metastatic osteosarcoma tissue and non-metastatic osteosarcoma samples. The results indicated that CCL5 mRNA expression was increased in the metastatic osteosarcoma tissue when compared with non-metastatic osteosarcoma tissue. A TMA of osteosarcoma tissues was used to investigate the correlation of CCL5 protein expression and clinicopathological features by IHC. The IHC results demonstrated that the expression of CCL5 in non-metastatic osteosarcoma tissue was either absent or at low levels; however, metastatic osteosarcoma tissues frequently exhibited high expression of CCL5. The overall survival rates and the metastasis-free survival rates in the CCL5 high expression group were significantly lower than the CCL5 low expression group. The current data demonstrates that high expression of CCL5 was associated with metastatic potential of osteosarcoma and is an independent predictor of poor prognosis. These results suggest that CCL5 may have an important role in the malignant behavior of osteosarcoma; however, the specific functional and molecular mechanisms require further investigation. CCL5 may be useful as a biomarker for osteosarcoma prognosis, and maybe a potential molecular target for osteosarcoma therapy.

\section{Acknowledgements}

The present study was supported by the Hubei Province Health and Family Planning Scientific Research Project (grant no. WJ2017M058).

\section{References}

1. Mirabello L, Troisi RJ and Savage SA: Osteosarcoma incidence and survival rates from 1973 to 2004: Data from the surveillance, epidemiology, and end results program. Cancer 115: 1531-1543, 2009.

2. Luetke A, Meyers PA, Lewis I and Juergens H: Osteosarcoma treatment-where do we stand? A state of the art review. Cancer Treat Rev 40: 523-532, 2014.

3. Schall TJ, Bacon K, Toy KJ and Goeddel DV: Selective attraction of monocytes and $\mathrm{T}$ lymphocytes of the memory phenotype by cytokine RANTES. Nature 347: 669-671, 1990.
4. Roth SJ, Carr MW and Springer TA: C-C chemokines, but not the $\mathrm{C}-\mathrm{X}-\mathrm{C}$ chemokines interleukin- 8 and interferon-gamma inducible protein-10, stimulate transendothelial chemotaxis of T lymphocytes. Eur J Immunol 25: 3482-3488, 1995.

5. de la Rosa G, Longo N, Rodríguez-Fernández JL, Puig-Kroger A, Pineda A, Corbí AL and Sánchez-Mateos P: Migration of human blood dendritic cells across endothelial cell monolayers: Adhesion molecules and chemokines involved in subset-specific transmigration. J Leukoc Biol 73: 639-649, 2003.

6. Taub DD, Sayers TJ, Carter CR and Ortaldo JR: Alpha and beta chemokines induce NK cell migration and enhance NK-mediated cytolysis. J Immunol 155: 3877-3888, 1995.

7. Kameyoshi Y, Dörschner A, Mallet AI, Christophers E and Schröder JM: Cytokine RANTES released by thrombin-stimulated platelets is a potent attractant for human eosinophils. J Exp Med 176: 587-592, 1992.

8. Conti P, Pang X, Boucher W, Letourneau R, Reale M, Barbacane RC, Thibault $\mathbf{J}$ and Theoharides TC: RANTES is a pro-inflammatory chemokine and chemoattracts basophil cells to extravascular sites. J Pathol 183: 352-358, 1997.

9. Ben-Baruch A: Inflammation-associated immune suppression in cancer: The roles played by cytokines, chemokines and additional mediators. Semin Cancer Biol 16: 38-52, 2006.

10. Moran CJ, Arenberg DA, Huang CC, Giordano TJ, Thomas DG, Misek DE, Chen G, Iannettoni MD, Orringer MB, Hanash S and Beer DG: RANTES expression is a predictor of survival in stage I lung adenocarcinoma. Clin Cancer Res 8: 3803-3812, 2002.

11. Vaday GG, Peehl DM, Kadam PA and Lawrence DM: Expression of CCL5 (RANTES) and CCR5 in prostate cancer. Prostate 66: 124-134, 2006.

12. Niwa Y, Akamatsu H, Niwa H, Sumi H, Ozaki Y and Abe A: Correlation of tissue and plasma RANTES levels with disease course in patients with breast or cervical cancer. Clin Cancer Res 7: 285-289, 2001.

13. Kim HK, Song KS, Park YS, Kang YH, Lee YJ, Lee KR, Kim HK, Ryu KW, Bae JM and Kim S: Elevated levels of circulating platelet microparticles, VEGF, IL-6 and RANTES in patients with gastric cancer: Possible role of a metastasis predictor. Eur J Cancer 39: 184-191, 2003.

14. Sugasawa H, Ichikura T, Kinoshita M, Ono S, Majima T, Tsujimoto H, Chochi K, Hiroi S, Takayama E, Saitoh D, et al: Gastric cancer cells exploit CD4 $4^{+}$cell-derived CCL5 for their growth and prevention of $\mathrm{CD}^{+}$cell-involved tumor elimination. Int J Cancer 122: 2535-2541, 2008.

15. Duell EJ, Casella DP, Burk RD, Kelsey KT and Holly EA: Inflammation, genetic polymorphisms in proinflammatory genes TNF-A, RANTES, and CCR5, and risk of pancreatic adenocarcinoma. Cancer Epidemiol Biomarkers Prev 15: 726-731, 2006.

16. Karnoub AE, Dash AB, Vo AP, Sullivan A, Brooks MW, Bell GW, Richardson AL, Polyak K, Tubo R and Weinberg RA: Mesenchymal stem cells within tumour stroma promote breast cancer metastasis. Nature 449: 557-563, 2007.

17. Livak KJ and Schmittgen TD: Analysis of relative gene expression data using real-time quantitative PCR and the 2(-Delta Delta C(T)) method. Methods 25: 402-408, 2001.

18. Arndt CA, Rose PS, Folpe AL and Laack NN: Common musculoskeletal tumors of childhood and adolescence. Mayo Clin Proc 87: 475-487, 2012.

19. Aldinucci D and Colombatti A: The inflammatory chemokine CCL5 and cancer progression. Mediators Inflamm 2014: 292376, 2014.

20. Huang CY, Fong YC, Lee CY, Chen MY, Tsai HC, Hsu HC and Tang CH: CCL5 increases lung cancer migration via PI3K, Akt and NF-kappaB pathways. Biochem Pharmacol 77: 794-803, 2009.

21. Borsig L, Wolf MJ, Roblek M, Lorentzen A and Heikenwalder M: Inflammatory chemokines and metastasis-tracing the accessory. Oncogene 33: 3217-3224, 2014.

22. Wang SW, Liu SC, Sun HL, Huang TY, Chan $\mathrm{CH}$, Yang CY, Yeh HI, Huang YL, Chou WY, Lin YM and Tang CH: CCL5/CCR5 axis induces vascular endothelial growth factor-mediated tumor angiogenesis in human osteosarcoma microenvironment. Carcinogenesis 36: 104-114, 2015.

23. Wang SW, Wu HH, Liu SC, Wang PC, Ou WC, Chou WY, Shen YS and Tang CH: CCL5 and CCR5 interaction promotes cell motility in human osteosarcoma. PLoS One 7: e35101, 2012.

24. Stormes KA, Lemken CA, Lepre JV, Marinucci MN and Kurt RA: Inhibition of metastasis by inhibition of tumor-derived CCL5. Breast Cancer Res Treat 89: 209-212, 2005. 